\title{
GENERAL UNCROSSING COVERING PATHS INSIDE THE AXIS-ALIGNED BOUNDING BOX
}

\author{
Marco Ripà \\ sPIqr Society, World Intelligence Network \\ Email : marcokrt1984@yahoo.it
}

\begin{abstract}
Given the finite set of $n_{1} \cdot n_{2} \cdots n_{k}$ points $G_{n_{1}, n_{2}, \ldots, n_{k}} \subset \mathbb{R}^{k}$ such that $n_{k} \geq$ $\ldots \geq n_{2} \geq n_{1} \in \mathbb{Z}^{+}$, we introduce a new algorithm, called $\mathrm{M} \Lambda \mathrm{I}$, which returns an uncrossing covering path inside the minimum axis-aligned bounding box $\left[0, n_{1}-1\right] \times$ $\left[0, n_{2}-1\right] \times \cdots \times\left[0, n_{k}-1\right]$, consisting of $3 \cdot \prod_{i=1}^{k-1} n_{i}-2$ links of prescribed length $n_{k}-1$ units. Thus, for any $n_{k} \geq 3$, the link length of the covering path provided by our M $\Lambda \mathrm{I}$-algorithm is smaller than the cardinality of the set $G_{n_{1}, n_{2}, \ldots, n_{k}}$. Furthermore, assuming $k>2$, we present an uncrossing covering path for $G_{3,3, \ldots, 3}$, comprising only $20 \cdot 3^{k-3}-2$ two units long edges, which is constrained by the axis-aligned bounding box $[0,4-\sqrt{3}] \times[0,4-\sqrt{3}] \times[0,2] \times \cdots \times[0,2]$.
\end{abstract}

Keywords: Path covering, M $\Lambda \mathrm{I}$-algorithm, AABB, Polygonal chain, Optimization problem, Link distance, Minimum bounding box, Analytical geometry.

\section{INTRODUCTION}

In the present paper we will study constrained optimization problems which are strongly related to three-dimensional integrated circuits (3D ICs) design, and could have applications in robot manufacturing. In order to compactly describe these fundamental problems, and extend our results to higher dimensions, we shall do well to begin with a few basic definitions.

Definition 1 Let $W$ and $Z$ be two sets, and let us denote the Cartesian product by symbol " $\times$ ". Thus, $W \times Z:=\{(w, z): w \in W \wedge z \in Z\}$, since it represents the set of all points $(w, z)$, where $w \in W$ and $z \in Z$.

Definition 2 Let $n_{1}, n_{2}, \ldots, n_{k-1}, n_{k} \in \mathbb{Z}^{+}$be such that $n_{1} \leq n_{2} \leq \ldots \leq n_{k-1} \leq n_{k}$. We define, $\quad \forall k \in \mathbb{Z}^{+}, \quad G_{n_{1}, n_{2}, \ldots, n_{k}}:=\left\{0,1, \ldots, n_{1}-1\right\} \times\left\{0,1, \ldots, n_{2}-1\right\} \times \cdots \times$ $\left\{0,1, \ldots, n_{k}-1\right\}$, so that the grid $G_{n_{1}, n_{2}, \ldots, n_{k}}$ is a set of $\prod_{i=1}^{k} n_{i}$ points in the Euclidean space $\mathbb{R}^{k}$.

Definition $3 \forall k \in \mathbb{Z}^{+}$, let us denote by $\hat{B}_{n_{1}, n_{2}, \ldots, n_{k}}:=\left\{\left(x_{1}, x_{2}, \ldots, x_{k}\right): x_{1} \in\left[0, n_{1}-1\right] \wedge\right.$ $\left.x_{2} \in\left[0, n_{2}-1\right] \wedge \ldots \wedge x_{k} \in\left[0, n_{k}-1\right]\right\} \subset \mathbb{R}^{k}$ the $M A A B B$ (i.e., minimum axis-aligned bounding box), and let us define $B_{n_{1}, n_{2}, \ldots, n_{k}}:=\left\{\left(x_{1}, x_{2}, \ldots, x_{k}\right): x_{1} \in\left[0, n_{1}\right] \wedge x_{2} \in\right.$ $\left.\left[0, n_{2}\right] \wedge \ldots \wedge x_{k} \in\left[0, n_{k}\right]\right\} \subset \mathbb{R}^{k}$ as $R A A B B$ (i.e., regular axis-aligned bounding box).

Definition 4 A covering path is a directed polygonal chain that visits every node of $G_{n_{1}, n_{2}, \ldots, n_{k}}$ exactly once, while a covering trail is a directed polygonal chain that joins every node of $G_{n_{1}, n_{2}, \ldots, n_{k}}$, but it can visit any of them more than once; in both cases, edges cannot be repeated 
and every couple of consecutive edges cannot be collinear. A covering path is uncrossing if none of its edges meets one other, and it is self-intersecting otherwise.

Definition 5 A covering circuit $F_{n_{1}, n_{2}, \ldots, n_{k}}$ is a closed directed trail that visits every node of $G_{n_{1}, n_{2}, \ldots, n_{k}}$ in which the starting point is equal to the endpoint. A covering cycle for $G_{n_{1}, n_{2}, \ldots, n_{k}}$ is a covering circuit in which the only repeated node can be the first/last one. In particular, we call regular covering cycle a cycle whose endpoint/starting point belongs to $G_{n_{1}, n_{2}, \ldots, n_{k}}$ and we call smart covering cycle a cycle whose endpoint/starting point is a Steiner point (i.e., a point that does not belong to $\left.G_{n_{1}, n_{2}, \ldots, n_{k}}\right)$.

Definition 6 The link length $h(Q)$ of a covering trail/circuit $Q_{n_{1}, n_{2}, \ldots, n_{k}}$ for $G_{n_{1}, n_{2}, \ldots, n_{k}}$ corresponds to the number of its edges, while the length of a given edge is the Euclidean distance between its two endpoints. Lastly, let us define the length classes of a covering trail/circuit as the set comprising all the lengths of its edges, denoting by $l_{1 \leq j \leq \prod_{i=1}^{k} n_{i}-1}(Q)$ the $j$-th element of the aforementioned set (where $l_{1}(Q)<l_{2}(Q)<\ldots<l_{j_{\max }}(Q)$ ) and simplify the notation by omitting the subscript if $l_{1}(Q)=l_{j_{\max }}(Q)$, so that $l(Q):=l_{1}(Q)$.

Thanks to the new M $\Lambda \mathrm{I}$-algorithm, described in Section II, we solve a pivotal problem concerning uncrossing covering paths consisting of less than $\prod_{i=1}^{k} n_{i}-1$ links of prescribed length [1], lying entirely inside the MAABB of any $k$-dimensional finite set of $n_{1} \times n_{2} \times \cdots$ $\times n_{k-1} \times n_{k}$ points.

In Section II, we also show how, for specific cases as $n_{1}=n_{k}=3$, it is possible to shorten the link length [2] of the general solution provided by the M $\Lambda \mathrm{I}$-algorithm if we consider the bounding box $\prod_{i=1}^{k}\left[0, n_{i}\right]$ (i.e., the RAABB) instead of the MAABB. Moreover, referring to the grid graphs $G_{3,3}=\{0,1,2\} \times\{0,1,2\}$ and $G_{3,3,3}=\{0,1,2\} \times\{0,1,2\} \times\{0,1,2\}$, we have constructively proved in [3] the existence of self-intersecting covering paths inside the MAABB (see Definition 3) that are formed by less than $3^{k}$ line segments, all belonging to the same irrational length class.

The aim of this work is to find valid solutions for some problems that arise when we combine in different ways a few basic constraints on the paths needed to cover all the points of the set $G_{n_{1}, n_{2}, \ldots, n_{k}}$ (see Definition 2) $[2,4]$.

The four fundamental constraints we are interested in, are as follows:

1. Visit all the points of $G_{n_{1}, n_{2}, \ldots, n_{k}}$ with an uncrossing covering path $P_{n_{1}, n_{2}, \ldots, n_{k}}$ [5];

2. Consider only covering paths such that all their edges belong to a unique length class $l\left(P_{n_{1}, n_{2}, \ldots, n_{k}}\right) \in \mathbb{R}$

3. $P_{n_{1}, n_{2}, \ldots, n_{k}} \subset B_{n_{1}, n_{2}, \ldots, n_{k}}$;

4. The link length of $P_{n_{1}, n_{2}, \ldots, n_{k}}$ has to be smaller than the cardinality of the set $G_{n_{1}, n_{2}, \ldots, n_{k}}$. Therefore let $h\left(P_{n_{1}, n_{2}, \ldots, n_{k}}\right)<\prod_{i=1}^{k} n_{i}$.

The additional constraint that will be considered is to replace the RAABB with the MAABB [3]. Thus, merely $P_{n_{1}, n_{2}, \ldots, n_{k}} \subseteq \widehat{B}_{n_{1}, n_{2}, \ldots, n_{k}}$, instead of $P_{n_{1}, n_{2}, \ldots, n_{k}} \subset B_{n_{1}, n_{2}, \ldots, n_{k}}$ (as stated by the third rule above). 
The goal to minimize the total (Euclidean) length of the covering path, denoted by $\lambda\left(P_{n_{1}, n_{2}, \ldots, n_{k}}\right)$, will not be taken into account in this paper [6,7], and it is trivial to note that it cannot be less than $\prod_{i=1}^{k} n_{i}-1$ units, which implies $l\left(P_{n_{1}, n_{2}, \ldots, n_{k}}\right)=1$. Consequently, we can be interested in reducing/minimizing the link length of $P_{n_{1}, n_{2}, \ldots, n_{k}}$ if and only if all the previously stated conditions have been fulfilled, so that $l\left(P_{n_{1}, n_{2}, \ldots, n_{k}}\right) \cdot h\left(P_{n_{1}, n_{2}, \ldots, n_{k}}\right)=$ $\lambda\left(P_{n_{1}, n_{2}, \ldots, n_{k}}\right)$.

Before asking if there exists any covering path which satisfies the four fundamental constraints, and if $l\left(P_{n_{1}, n_{2}, \ldots, n_{k}}\right)$ is unique or not for some $k$-tuple $\left(n_{1}, n_{2}, \ldots, n_{k}\right)$, let us give a valid lower bound on the link length of any covering trail for $G_{n_{1}, n_{2}, \ldots, n_{k}}$.

Since Reference [5], Equation 4, guarantees a lower bound for the link length of the minimal covering trail (without additional constraints as above), and considering that, by definition, it cannot be greater than the link length of the minimal covering path, $\forall k, n \in \mathbb{N}-\{0,1,2\}$ we have

$$
h\left(P_{n_{1}, n_{2}, \ldots, n_{k}}\right) \geq\left\lceil 3 \cdot \frac{\prod_{i=1}^{k} n_{i}-\sum_{i=1}^{k-2} n_{i}+k-3}{2 \cdot n_{k}+n_{k-1}-3}\right\rceil+k-2 .
$$

\section{THE MAI-ALGORITHM}

We introduce the general $\mathrm{M} \Lambda \mathrm{I}$-algorithm, which is able to cover $G_{n_{1}, n_{2}, \ldots, n_{k}}$ for any given $k$-tuple $\left(n_{1}, n_{2}, \ldots, n_{k-1}, n_{k}\right)$ such that $k \geq 2$, under the four fundamental constraints stated in the previous section, plus the additional one. The resulting, inside the MAABB, uncrossing covering path has a total of $\prod_{i=1}^{k-1} n_{i}+2 \cdot\left(\left(\prod_{i=1}^{k-1} n_{i}\right)-1\right)$ edges.

Hence,

$$
h\left(P_{n_{1}, n_{2}, \ldots, n_{k}}\right)=3 \cdot \prod_{i=1}^{k-1} n_{i}-2 .
$$

Since $k \geq 2$, the inequality

$$
3 \cdot \prod_{i=1}^{k-1} n_{i}-2<\prod_{i=1}^{k} n_{i}
$$

holds for any $n_{1} \leq n_{2} \leq \ldots \leq n_{k-1} \leq n_{k}$ such that $n_{k}>2$.

Let $n_{1}>1$. If $n_{k}=2$, it is clearly possible to join the $2^{k}$ vertices of the grid spending $2^{k}-$ 1 links of unitary length, as explained in [6], reproducing $2^{k-2}$ times the trivial twodimensional covering path $P_{2,2}=(0,0)-(0,1)-(1,1)-(1,0)$ and fixing the aforementioned 3 . $2^{k-2}$ edges with $2^{k-2}-1$ additional links. Similarly, if $k=1$, then $P_{n_{1}}=(0,0)-\left(0, n_{1}\right) \equiv \widehat{B}_{n_{1}}$ always works.

Thus, from here on, let us assume $3 \leq n_{k} \wedge k \geq 2$, and the M $\Lambda \mathrm{I}$-algorithm can always be applied, providing a valid path such that $h\left(P_{n_{1}, n_{2}, \ldots, n_{k}}\right)+1<\prod_{i=1}^{k} n_{i}$.

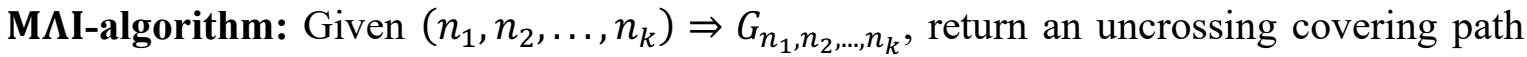
$P_{n_{1}, n_{2}, \ldots, n_{k}} \subseteq \widehat{B}_{n_{1}, n_{2}, \ldots, n_{k}} \subset B_{n_{1}, n_{2}, \ldots, n_{k}}$ such that $h\left(P_{n_{1}, n_{2}, \ldots, n_{k}}\right)=3 \cdot \prod_{i=1}^{k-1} n_{i}-2$ and $l(P)=$ $n_{k}-1$. 
$P_{n_{1}, n_{2}, \ldots, n_{k}}$ [first layer] $=(0,0,0, \ldots, 0,0)-\left(0,0,0, \ldots, 0, n_{k}-1\right)-\left(\frac{1}{2}, 0,0, \ldots, 0, n_{k}-1-\right.$

$\left.\sqrt{\left(n_{k}-1\right)^{2}-\frac{1}{4}}\right)-\left(1,0,0, \ldots, 0, n_{k}-1\right)-(1,0,0, \ldots, 0,0)-\left(\frac{3}{2}, 0,0, \ldots, 0, \sqrt{\left(n_{k}-1\right)^{2}-\frac{1}{4}}\right)-$

$(2,0,0, \ldots, 0,0)-\left(2,0,0, \ldots, 0, n_{k}-1\right)-\left(\frac{5}{2}, 0,0, \ldots, 0, n_{k}-1-\sqrt{\left(n_{k}-1\right)^{2}-\frac{1}{4}}\right)-$

$\left(3,0,0, \ldots, 0, n_{k}-1\right)-(3,0,0, \ldots, 0,0)-\left(\frac{7}{2}, 0,0, \ldots, 0, \sqrt{\left(n_{k}-1\right)^{2}-\frac{1}{4}}\right)-\ldots$ and so forth, until either $\left(n_{1}-1,0,0, \ldots, 0, n_{k}-1\right)$ or $\left(n_{1}-1,0,0, \ldots, 0,0\right)$ is reached last (depending on whether $n_{1}$ is odd or even, respectively).

Now, let us assume that $n_{1}$ is even, so that the last link to cover the first layer is $\left(n_{1}-1,0,0, \ldots, 0, n_{k}-1\right)-\left(n_{1}-1,0,0, \ldots, 0,0\right)$. Otherwise, $n_{1}$ have to be odd and we fall in the opposite case, where two flipped bridges will alternately be switched moving from layer to layer.

Then, by assuming for simplicity $n_{1}: n_{1}=2 \cdot m$, where $m \in \mathbb{N}-\{0,1\}$, we have

$P_{n_{1}, n_{2}, \ldots, n_{k}}\left[\right.$ first bridge] $=\left(n_{1}-1, \frac{1}{2}, 0, \ldots, 0, \sqrt{\left(n_{k}-1\right)^{2}-\frac{1}{4}}\right)-\left(n_{1}-1,1,0, \ldots, 0,0\right)$.

$P_{n_{1}, n_{2}, \ldots, n_{k}}[$ second layer $]=\left(n_{1}-1,1,0, \ldots, 0, n_{k}-1\right)-\left(n_{1}-1-\frac{1}{2}, 1,0, \ldots, 0, n_{k}-1-\right.$ $\left.\sqrt{\left(n_{k}-1\right)^{2}-\frac{1}{4}}\right)-\left(n_{1}-2,1,0, \ldots, 0, n_{k}-1\right)-\left(n_{1}-2,1,0, \ldots, 0,0\right)-\left(n_{1}-2-\frac{1}{2}, 1,0, \ldots, 0\right.$, $\left.\sqrt{\left(n_{k}-1\right)^{2}-\frac{1}{4}}\right)-\left(n_{1}-3,1,0, \ldots, 0,0\right)-\left(n_{1}-3,1,0, \ldots, 0, n_{k}-1\right)-\ldots$ and so on. In this way, we finally reach $(0,0,1, \ldots, 0,0)$, the last point of the second layer.

$P_{n_{1}, n_{2}, \ldots, n_{k}}$ [second bridge] $=\left(0,0, \frac{3}{2}, \ldots, 0, \sqrt{\left(n_{k}-1\right)^{2}-\frac{1}{4}}\right)-(0,0,2, \ldots, 0,0)$.

We repeat the same pattern until we reach the endpoint, which is the last visited point among all the elements of the set $V$ defined as

$$
\begin{gathered}
V:=\left\{\left(x_{1}, x_{2}, \ldots, x_{k-1}, x_{k}\right): x_{1} \in\left\{0, n_{1}-1\right\} \wedge x_{2} \in\left\{0, n_{2}-1\right\} \wedge \ldots \wedge x_{k-1} \in\right. \\
\left.\left\{0, n_{k-1}-1\right\} \wedge x_{k} \in\left\{0, n_{k}-1\right\}\right\} .
\end{gathered}
$$

Therefore, $\forall\left(n_{1}, n_{2}, \ldots, n_{k}\right): n_{1} \leq n_{2} \leq \ldots \leq n_{k} \in \mathbb{N}-\{0,1\}, \exists P_{n_{1}, n_{2}, \ldots, n_{k}}$, an inside the MAABB uncrossing covering path, such that $h\left(P_{n_{1}, n_{2}, \ldots, n_{k}}\right)<\prod_{i=1}^{k} n_{i} \wedge l\left(P_{n_{1}, n_{2}, \ldots, n_{k}}\right)=$ $n_{k}-1$. Moreover, the M $\Lambda \mathrm{I}$-algorithm let $P_{n_{1}, n_{2}, \ldots, n_{k}}$ join an increasing number of points with every triplet of consecutive edges as $n_{k}$ grows, and Equations (4)\&(5) show how its efficiency becomes absolute when $n_{k}$ approaches infinity. Then from Equation (2), it follows that

$$
\frac{h\left(P_{n_{1}, n_{2}, \ldots, n_{k}}\right)}{\prod_{i=1}^{k} n_{i}}=\frac{3}{n_{k}}-\frac{2}{\prod_{i=1}^{k} n_{i}} .
$$

Hence,

$$
\lim _{n_{k} \rightarrow+\infty} \frac{h\left(P_{n_{1}, n_{2}, \ldots, n_{k}}\right)}{\prod_{i=1}^{k} n_{i}}=0
$$




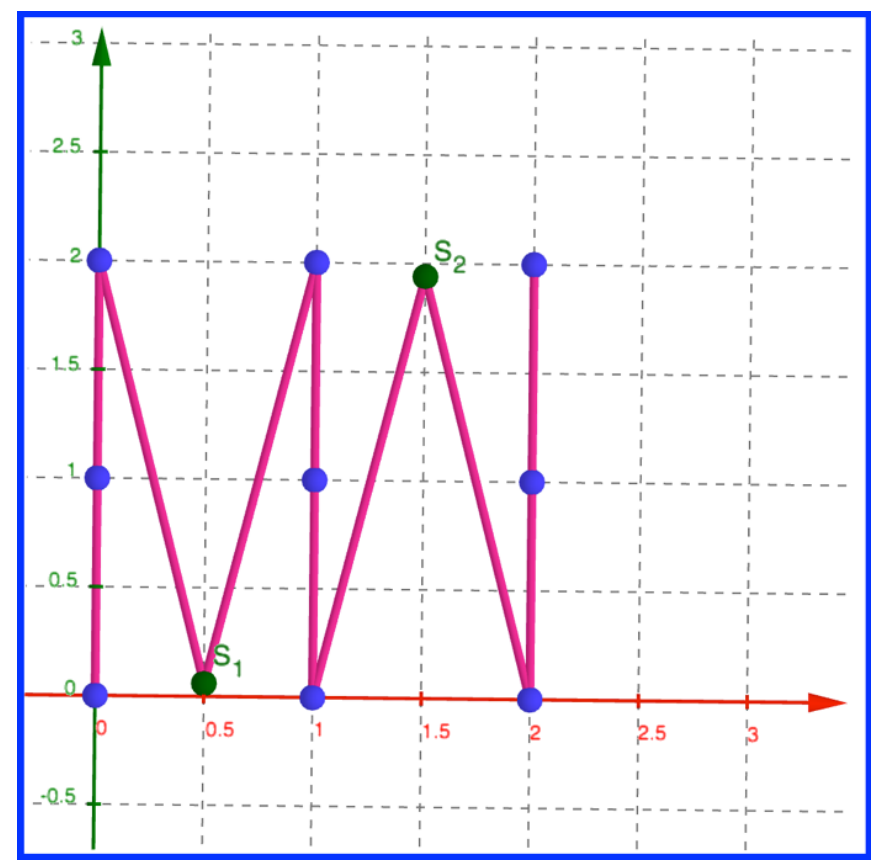

Figure 1. First layer of the covering path $P_{3,3,3}$, edges 1 to 7 : all of them belong to the length class 2 . The two Steiner points (in green) are $S_{1} \equiv\left(\frac{1}{2}, 2-\frac{\sqrt{15}}{2}\right) \cong(0.5,0.063508)$ and $\mathrm{S}_{2} \equiv\left(\frac{3}{2}, \frac{\sqrt{15}}{2}\right) \cong(1.5,1.936492)$ (picture realized with GeoGebra [8]).

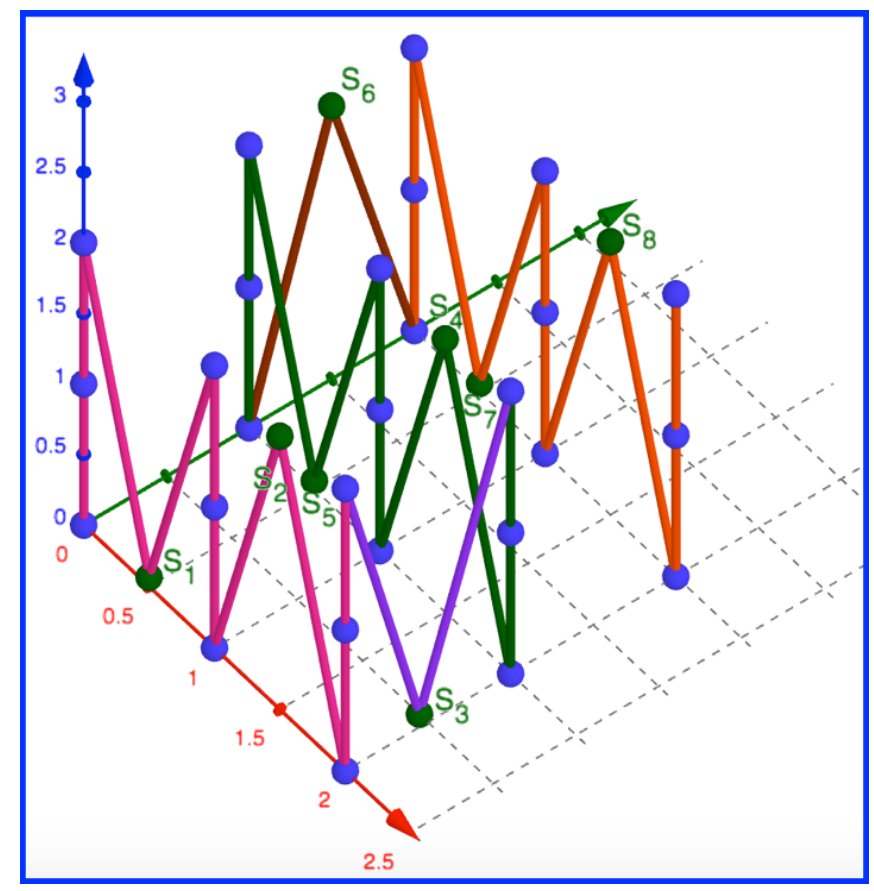

Figure 2. The whole directed covering path $P_{3,3,3}$, edges 1 to 25 : all of them belong to the length class 2 (picture realized with GeoGebra). 
As a couple of examples, we can take $P_{3,3} \subset \hat{B}_{3,3}$ and $P_{3,3,3} \subset \widehat{B}_{3,3,3}$, where $P_{3,3}=(0,0)-(0,2)-\left(\frac{1}{2}, 2-\frac{\sqrt{15}}{2}\right)-(1,2)-(1,0)-\left(\frac{3}{2}, \frac{\sqrt{15}}{2}\right)-(2,0)-(2,2) \quad$ and $P_{3,3,3}=(0,0,0)-$ $(0,0,2)-\left(\frac{1}{2}, 0,2-\frac{\sqrt{15}}{2}\right)-(1,0,2)-(1,0,0)-\left(\frac{3}{2}, 0, \frac{\sqrt{15}}{2}\right)-(2,0,0)-(2,0,2)-\left(2, \frac{1}{2}, 2-\frac{\sqrt{15}}{2}\right)-$

$(2,1,2)-(2,1,0)-\left(\frac{3}{2}, 1, \frac{\sqrt{15}}{2}\right)-(1,1,0)-(1,1,2)-\left(\frac{1}{2}, 1,2-\frac{\sqrt{15}}{2}\right)-(0,1,2)-(0,1,0)-\left(0, \frac{3}{2}, \frac{\sqrt{15}}{2}\right)-$ $(0,2,0)-(0,2,2)-\left(\frac{1}{2}, 2,2-\frac{\sqrt{15}}{2}\right)-(1,2,2)-(1,2,0)-\left(\frac{3}{2}, 2, \frac{\sqrt{15}}{2}\right)-(2,2,0)-(2,2,2)$, as shown in Figures $1 \& 2$.

Referring to $G_{3,3}$ and $G_{3,3,3}$ (as above), it is possible to show also the existence of selfintersecting covering paths, $M_{3,3} \subset \widehat{B}_{3,3}$ and $M_{3,3,3} \subset \widehat{B}_{3,3,3}$, such that their link length is $\prod_{i=1}^{k} 3^{i}-1$ and $l\left(M_{3,3}\right)=l\left(M_{3,3,3}\right)=\sqrt{5}>n_{k}-1$. In particular, as shown in Figure 3, $M_{3,3}=(1,2)-(2,0)-(0,1)-(2,2)-(1,0)-(0,2)-(2,1)-(0,0)-\left(\sqrt{\frac{5}{2}}, \sqrt{\frac{5}{2}}\right), \quad$ while $\quad M_{3,3,3}=$ $(2,0,0)-(0,1,0)-(2,2,0)-(1,0,0)-(0,2,0)-(1,2,2)-(0,0,2)-(2,1,2)-(0,2,2)-(1,0,2)-$ $(2,2,2)-(0,1,2)-(2,0,2)-(2,2,1)-(0,1,1)-(2,0,1)-(1,2,1)-(0,0,1)-(2,1,1)-(0,2,1)-$ $(1,0,1)-(1,2,0)-(1,1,2)-(2,1,0)-(0,0,0)-\left(\sqrt{\frac{5}{2}}, \sqrt{\frac{5}{2}}, 0\right)-\left(\frac{\sqrt{10}}{12} \cdot(6-\sqrt{24-3 \cdot \sqrt{10}})\right.$, $\left.\frac{\sqrt{10}}{12} \cdot(6-\sqrt{24-3 \cdot \sqrt{10}}), \frac{1}{2} \cdot \sqrt{\frac{5}{3} \cdot(4+\sqrt{10})}\right)$ (see [3], proof of Theorem 2, for details).

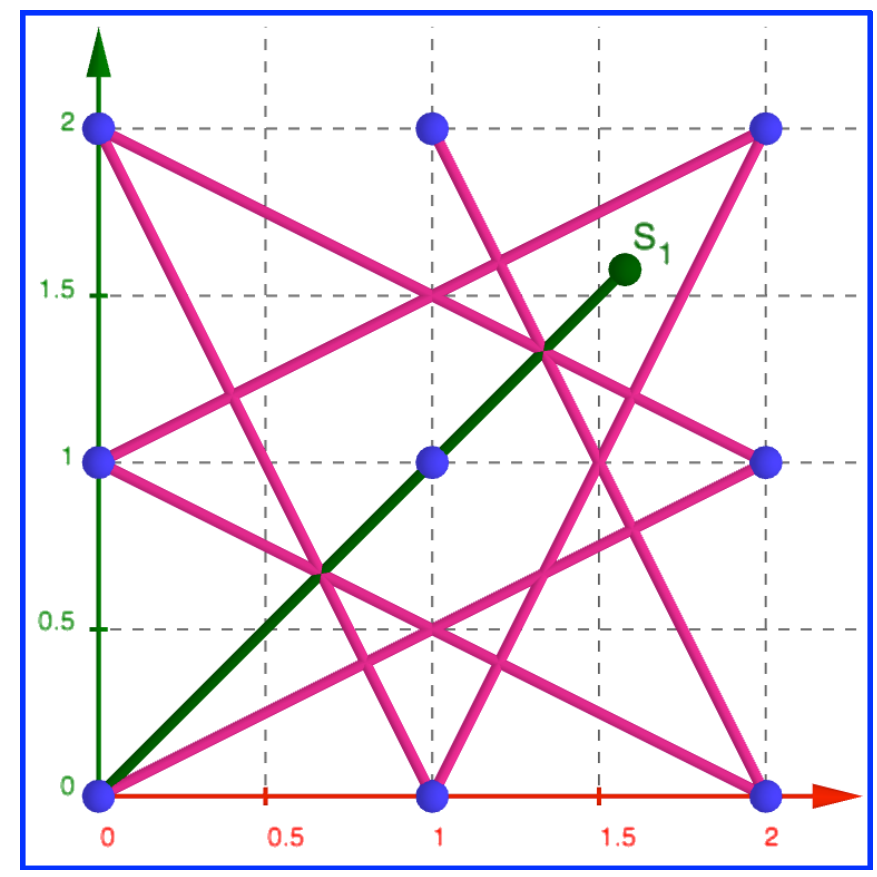

Figure 3. The self-intersecting covering path $M_{3,3}$ consists of 8 edges, and all of them belong to the length class $\sqrt{5}$ (picture realized with GeoGebra).

On the other hand, considering all the grids $G_{3,3, \ldots, 3}(k): k \geq 2$, if we loosen the constraint on the minimum axis-aligned bounding box to the regular one (or at least to $\breve{B}_{3,3, \ldots, 3}:=$ $\left.[0,2] \times[0,4-\sqrt{3}] \times[0,4-\sqrt{3}] \times \prod_{i=1}^{k-3}[0,2]\right)$, we can easily show the existence of 
uncrossing covering paths with at most $h\left(\check{P}_{3,3, \ldots, 3}\right)=20 \cdot 3^{k-3}-2$ links, all belonging to the length class $l\left(\breve{P}_{3,3, \ldots, 3}\right)=2$ (see Figures $\left.4 \& 5\right)$.

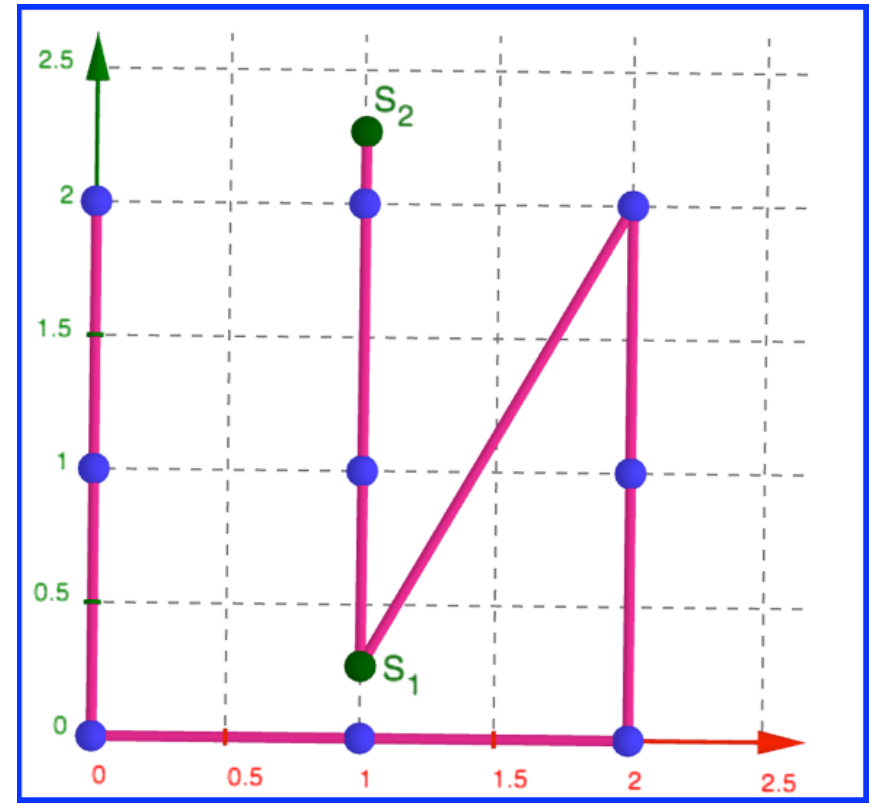

Figure 4. The covering path $\breve{P}_{3,3}=(0,2)-(0,0)-(2,0)-(2,2)-(1,2-\sqrt{3})-(1,4-\sqrt{3})$ (picture realized with GeoGebra).

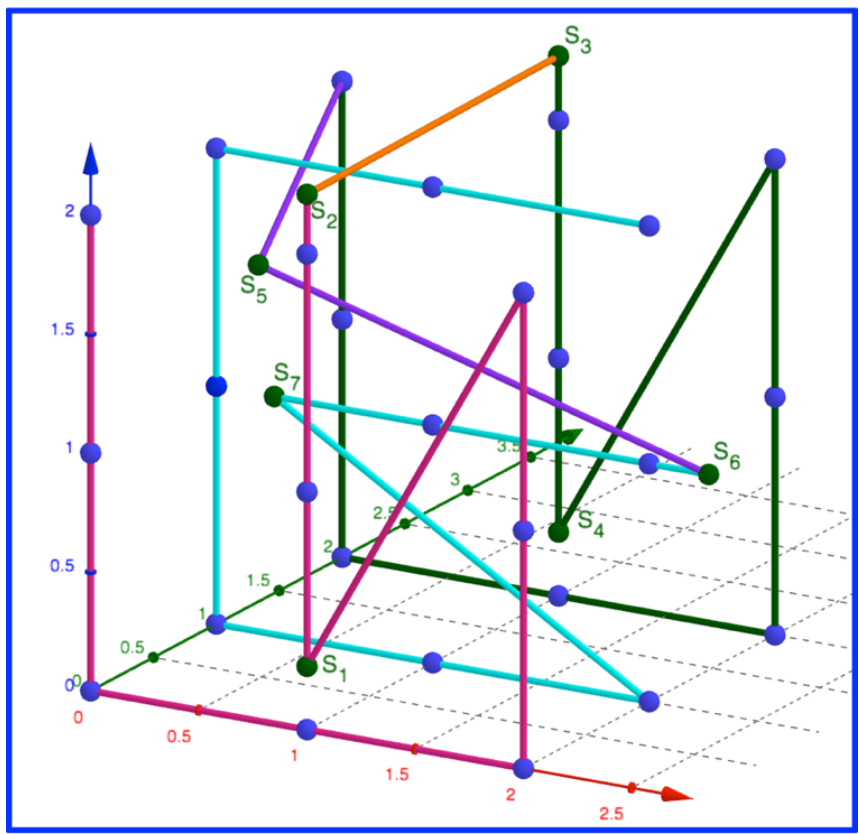

Figure 5. The (uncrossing) covering path $\breve{P}_{3,3,3}=(0,2,0)-(0,0,0)-(2,0,0)-(2,2,0)-(1,2-\sqrt{3}, 0)-$

$$
\left(\frac{7}{10}, \frac{(33 \cdot \sqrt{3}-37+\sqrt{2 \cdot(2541 \cdot \sqrt{3}-4247)})}{20}, \frac{(33 \cdot \sqrt{3}-37-\sqrt{2 \cdot(2541 \cdot \sqrt{3}-4247)})}{20}\right)-(4-\sqrt{3}, 1,1)-(2-\sqrt{3}, 1,1)-
$$

$(2,0,1)-(0,0,1)-(0,2,1)-(2,2,1)$ extends $\breve{P}_{3,3}$ to three dimensions [8]. 
Consider the uncrossing covering path $\check{P}_{3,3,3}$ (see Figure 5 where $x_{\mathrm{S}_{5}}=0.7$ and the bigger of the two values of $y\left(x_{S_{5}}\right)$ have been selected). The Steiner point $S_{5} \equiv\left(x_{S_{5}}, y_{S_{5}}, z_{S_{5}}\right)$ can be arbitrarily chosen among many of the solutions provided by the intersection of the two spheres of radius 2 units which are centered in $\mathrm{A} \equiv(0,2,2)$ and $\mathrm{B} \equiv(4-\sqrt{3}, 1,1)$. Thus,

$$
\left\{\begin{array}{c}
x^{2}+(y-2)^{2}+(z-2)^{2}=2^{2} \\
(x-4+\sqrt{3})^{2}+(y-1)^{2}+(z-1)^{2}=2^{2}
\end{array} .\right.
$$

At this purpose, it is necessary to preliminary point out that the two conditions $y_{\mathrm{S}_{5}} \in$ $\left[\frac{3}{2}-\sqrt{\frac{466 \cdot \sqrt{3}-333}{249}}, 4-\sqrt{3}\right]-\{2\}$ and $z_{S_{5}} \leq 2$ should be satisfied, and they are a sufficient restriction to ensure that any constrained solution of (7) returns a valid $S_{5}$, avoiding any selfintersecting risk for $\breve{P}_{3,3,3}$ (see Relations (7) to (9) below), so we have

$$
\begin{gathered}
y_{S_{5}}=\frac{1}{4} \cdot\left(\sqrt{4 \cdot(8 \cdot \sqrt{3}-21) \cdot x^{2}+4 \cdot(108-53 \cdot \sqrt{3}) \cdot x+336 \cdot \sqrt{3}-601}+2 \cdot(4-\sqrt{3}) \cdot x+8 \cdot \sqrt{3}-13\right) \wedge \\
z_{S_{5}}=\frac{1}{4} \cdot\left(-\sqrt{4 \cdot(8 \cdot \sqrt{3}-21) \cdot x^{2}+4 \cdot(108-53 \cdot \sqrt{3}) \cdot x+336 \cdot \sqrt{3}-601}+2 \cdot(4-\sqrt{3}) \cdot x+8 \cdot \sqrt{3}-13\right) .
\end{gathered}
$$

More specifically, the circumference by (6) under the pair of previously stated conditions assures the existence of (at least) one possible Steiner point $S_{5}$, which is well-defined assuming $\left.S_{5} \equiv \bar{S}_{5} \vee\left(y_{S_{5}} \geq z_{S_{5}}\right) \wedge S_{5} \not \equiv \bar{S}_{5}\right)$ (as above), for any

$$
x \in\left[2-\frac{\sqrt{3}}{2}-\sqrt{\frac{87+128 \cdot \sqrt{3}}{498}}, \frac{65 \cdot(2-\sqrt{3})-\sqrt{916 \cdot \sqrt{3}-1549}}{8 \cdot(5-2 \cdot \sqrt{3})}\right] \approx[0.346647,0.918696] \text {. }
$$

Forasmuch as $\overline{\overline{\mathrm{S}}}_{5} \equiv\left(\frac{53 \cdot \sqrt{3}-108+\sqrt{208 \cdot \sqrt{3}-313}}{8 \cdot(2 \cdot \sqrt{3}-5)}, 2, \frac{151-2 \cdot \sqrt{3}-\sqrt{20092 \cdot \sqrt{3}-17383}}{104}\right)$ cannot be accepted as a solution in order to generate a covering path which is not self-intersecting (i.e., $y_{S_{5}} \neq 2$ by one of the stated conditions), let us replace the invalid Steiner point $\overline{\bar{S}}_{5}$ with $\overline{\mathrm{S}}_{5} \equiv$ $\left(\frac{53 \cdot \sqrt{3}-108+\sqrt{208 \cdot \sqrt{3}-313}}{8 \cdot(2 \cdot \sqrt{3}-5)}, \frac{151-2 \cdot \sqrt{3}-\sqrt{20092 \cdot \sqrt{3}-17383}}{104}, 2\right)$. Finally, we observe that there are not any other critical values, because (see Figure 6, looking for any possible collision between the twelfth link of $\breve{P}_{3,3,3}$ and the light blue layer in the middle)

$$
\begin{gathered}
\left\{\begin{array} { c } 
{ x ^ { 2 } + ( y - 2 ) ^ { 2 } + ( z - 2 ) ^ { 2 } = r ^ { 2 } } \\
{ ( x - 4 + \sqrt { 3 } ) ^ { 2 } + ( y - 1 ) ^ { 2 } + ( z - 1 ) ^ { 2 } = r ^ { 2 } }
\end{array} \cap \left\{\begin{array}{c}
\frac{x}{2-\sqrt{3}}=2-y \\
y=z
\end{array} \Rightarrow\right.\right. \\
\Rightarrow\left\{\begin{array} { c } 
{ x ^ { 2 } + ( y - 2 ) ^ { 2 } + ( z - 2 ) ^ { 2 } = r ^ { 2 } } \\
{ ( x - 4 + \sqrt { 3 } ) ^ { 2 } + ( y - 1 ) ^ { 2 } + ( z - 1 ) ^ { 2 } = r ^ { 2 } } \\
{ \frac { x } { 2 - \sqrt { 3 } } = 2 - y } \\
{ y = z }
\end{array} \Rightarrow \left\{\begin{array}{c}
r=\frac{\sqrt{94725-21288 \cdot \sqrt{3}}}{122} \\
x=\frac{192-85 \cdot \sqrt{3}}{122} \\
y=\frac{115-22 \cdot \sqrt{3}}{122} \\
z=\frac{115-22 \cdot \sqrt{3}}{122}
\end{array} \Rightarrow r<2 .\right.\right.
\end{gathered}
$$




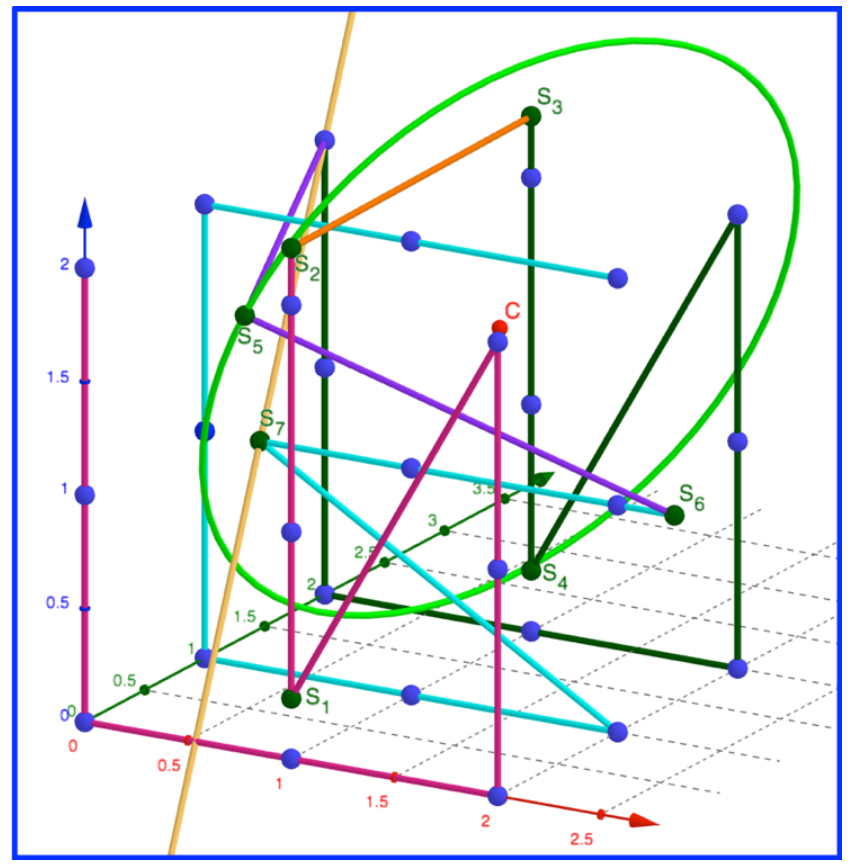

Figure 6. Graphical proof that no collision will occur in the process of moving $S_{5}$ along the allowed $\operatorname{arc}\left(2>y_{\mathrm{S}_{5}} \geq z_{\mathrm{S}_{5}}\right)$ of the circle given by Equation (6), as shown by comparison of (6) with (9) (i.e., observing that $2>r \approx 1.9715304811)$. The radius of the circle centered in $\mathrm{C} \equiv\left(\frac{4-\sqrt{3}}{2}, \frac{3}{2}, \frac{3}{2}\right)$ is $a=$ $\frac{\sqrt{8 \cdot \sqrt{3}-5}}{2} \approx 1.4879857577$, while a collision between the 13 th and the 15 th link would only occur for

$$
y_{\mathrm{S}_{5}}=\frac{161+2 \cdot \sqrt{3}-\sqrt{20092 \cdot \sqrt{3}-17383}}{104}<1=z_{\mathrm{S}_{5}}[8] .
$$

Let us extend the results to higher dimensions, we then assume $k \geq 3$.

From Equations (7)\&(8), since it is possible to set an unlimited number of layer bridges by choosing distinct Steiner points as $\mathrm{S}_{5}$, we are able to straightforwardly derive uncrossing covering paths $\breve{P}_{3,3, \ldots, 3}$ with $18 \cdot 3^{k-3}+\sum_{j=0}^{k-4} 3^{j} \cdot 4=20 \cdot 3^{k-3}-2$ edges if we simply lift, by iteration, the given 3D pattern to the next dimension (in a very similar way as we previously shown in Figure 2 for the $\mathrm{M} \Lambda \mathrm{I}$-algorithm). Furthermore, referring to the same AABB (i.e., $\left.\breve{B}_{3,3, \ldots, 3}\right)$ and pattern by Figures $5 \& 6$, it is possible to save one more line for any dimensional bridge if we switch from uncrossing covering paths to covering trails, spending a total of $18 \cdot 3^{k-3}+\sum_{j=1}^{k-3} 3^{j}=\frac{13 \cdot 3^{k-2}-3}{2}$ links (at most) to join all the given $3^{k}$ points, and this consideration would suffice to reveal how many unexplored optimization problems could be studied starting from a different set of preliminary assumptions, rather than the four fundamental constraints stated in Section I.

We could also go further and decide (as a random example) to cover the grid with an unconstrained circuit of minimum link length, disregarding the RAABB, so we may accept $F_{2,2,2}=\left(-\frac{1+\sqrt{13}}{6},-\frac{1+\sqrt{13}}{6}, 0\right)-\left(\frac{7+\sqrt{13}}{6}, \frac{7+\sqrt{13}}{6}, 0\right)-\left(-\frac{1+\sqrt{13}}{4}, \frac{1}{2}, \frac{3+\sqrt{13}}{4}\right)$ $\left(\frac{7+\sqrt{13}}{6},-\frac{1+\sqrt{13}}{6}, 0\right)-\left(-\frac{1+\sqrt{13}}{6}, \frac{7+\sqrt{13}}{6}, 0\right)-\left(\frac{5+\sqrt{13}}{4}, \frac{1}{2}, \frac{3+\sqrt{13}}{4}\right)-\left(-\frac{1+\sqrt{13}}{6},-\frac{1+\sqrt{13}}{6}, 0\right) \quad$ and $F_{2,2,2}^{\prime}=(1-\sqrt{2}, 1-\sqrt{2}, 0)-(\sqrt{2}, \sqrt{2}, 0)-\left(\frac{1}{2}, \frac{1}{2}, 2 \cdot \sqrt{3}-\sqrt{\frac{3}{2}}\right)-(\sqrt{2}, 1-\sqrt{2}, 0)-(1-\sqrt{2}$ 
$\sqrt{2}, 0)-\left(\frac{1}{2}, \frac{1}{2}, 2 \cdot \sqrt{3}-\sqrt{\frac{3}{2}}\right)-(1-\sqrt{2}, 1-\sqrt{2}, 0)$ as a pair of valid solutions (see Figures $7 \& 8)$, while we may reject $(0,0,0)-(1,0,0)-(1,1,0)-(0,1,0)-(0,1,1)-(1,1,1)-(1,0,1)$ $(0,0,1)$ for two different reasons.

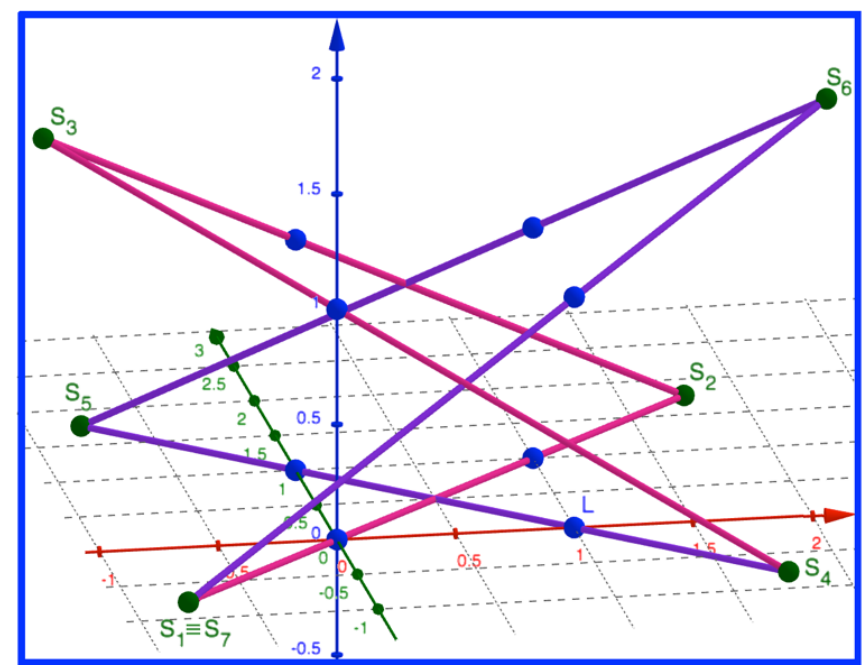

Figure 7. A smart covering cycle for $G_{2,2,2} \cdot F_{2,2,2}$ has link length of 6 , is constrained by $\left[-\frac{1+\sqrt{13}}{4}, \frac{5+\sqrt{13}}{4}\right] \times\left[-\frac{1+\sqrt{13}}{6}, \frac{7+\sqrt{13}}{6}\right] \times\left[0, \frac{3+\sqrt{13}}{4}\right] \nsubseteq \mathrm{RAABB}$, and is characterized by $l\left(F_{2,2,2}\right)=\frac{\sqrt{2} \cdot(4+\sqrt{13})}{3}($ see Definitions 3 to 6$)[8]$.

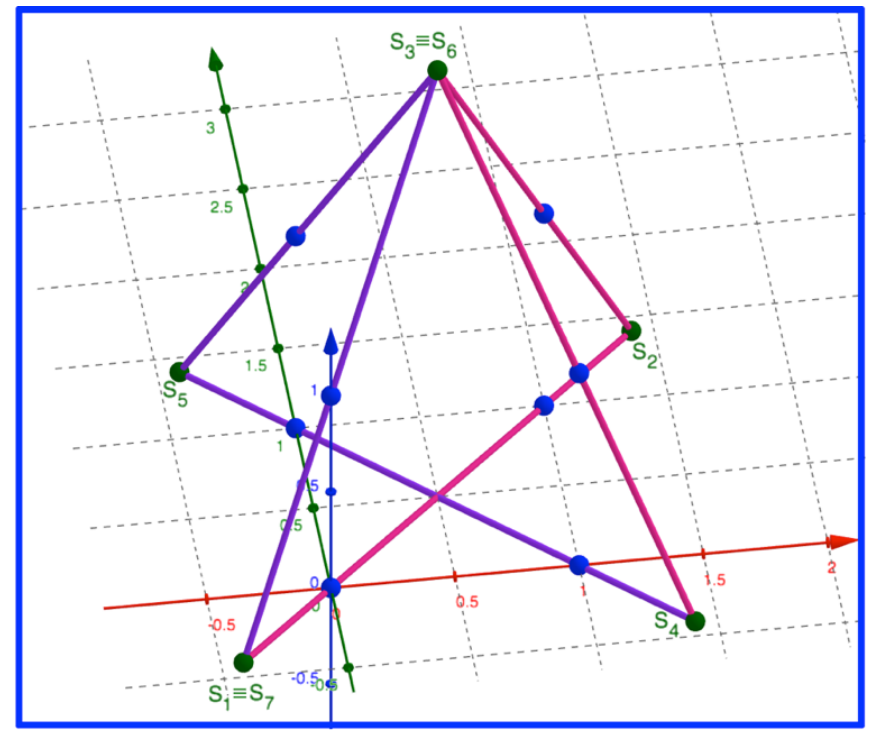

Figure 8. A covering circuit with optimal link length $h\left(F_{2,2,2}^{\prime}\right)=6$.

$F^{\prime}{ }_{2,2,2}$ is inside the AABB $[1-\sqrt{2}, \sqrt{2}] \times[1-\sqrt{2}, \sqrt{2}] \times\left[0,2 \cdot \sqrt{3}-\sqrt{\frac{3}{2}}\right] \nsubseteq \mathrm{RAABB}$ and is characterized by $l\left(F_{2,2,2}^{\prime}\right)=4-\sqrt{2}$ (see Definitions 3 to 6) [8].

Lastly, it is even possible to approach some optimization problems of path covering focusing on the AABB volume, such as finding the uncrossing covering path of minimal link length which subtends the minimum volume AABB. 
Let $G_{2,2,2}$ be given and assume $x_{\mathrm{S}_{1}}>0$. We could begin with the set of self-intersecting covering paths $\bar{P}_{2,2,2}\left(x_{\mathrm{S}_{1}}\right)=(0,1,0)-(0,0,0)-\left(x_{\mathrm{S}_{1}}, 0, x_{\mathrm{S}_{1}}\right)-\left(\frac{1}{2}, y_{\mathrm{S}_{2}}\left(x_{\mathrm{S}_{1}}\right), \frac{1}{2}\right)-\left(1-x_{\mathrm{S}_{1}}, 0\right.$, $\left.x_{S_{1}}\right)-(1,0,0)-(1,1,0)$, and then determine the value of $x_{S_{1}}$ that minimizes the volume of the AABB from

$$
\left\{\begin{array}{l}
\left(1-x_{\mathrm{S}_{1}}\right) \cdot y_{\mathrm{S}_{2}}+x_{\mathrm{S}_{1}}=\frac{1}{2} \\
\frac{\partial\left(\left(2 \cdot x_{\mathrm{S}_{1}}-1\right) \cdot y_{\mathrm{S}_{2}} \cdot x_{\mathrm{S}_{1}}\right)}{\partial x_{\mathrm{S}_{1}}}=0 \\
x_{\mathrm{S}_{1}}>1
\end{array}\right.
$$

Hence,

$$
\left\{\begin{array} { c } 
{ y _ { \mathrm { S } _ { 2 } } = \frac { 1 - 2 \cdot x _ { \mathrm { S } _ { 1 } } } { 2 \cdot ( 1 - x _ { \mathrm { S } _ { 1 } } ) } } \\
{ x _ { \mathrm { S } _ { 1 } } { } ^ { 3 } - 2 \cdot x _ { \mathrm { S } _ { 1 } } { } ^ { 2 } + x _ { \mathrm { S } _ { 1 } } = \frac { 1 } { 8 } } \\
{ x _ { \mathrm { S } _ { 1 } } > 1 }
\end{array} \Rightarrow \left\{\begin{array}{c}
x_{\mathrm{S}_{1}}=\frac{3+\sqrt{5}}{4} \\
y_{\mathrm{S}_{2}}=\frac{3+\sqrt{5}}{2}
\end{array}\right.\right.
$$

Now, let $\varphi:=\frac{1+\sqrt{5}}{2}$ (i.e., the well-known golden ratio).

It follows that $x_{\mathrm{S}_{1}}=\frac{1+\varphi}{2} \wedge y_{\mathrm{S}_{2}}=1+\varphi$.

Thus, for any $0<\varepsilon<\frac{\varphi-1}{2}$, we can easily derive the uncrossing covering path $\overline{\bar{P}}_{2,2,2}(\varepsilon)=$ $(0,1,0)-(0,0,0)-\left(\frac{1+\varphi}{2}, 0, \frac{1+\varphi}{2}\right)-\left(\frac{1}{2}, 1+\varphi, \frac{1}{2}\right)-\left(\frac{1-\varphi}{2}+\varepsilon, 2 \cdot \varphi \cdot \varepsilon, \frac{1+\varphi}{2}-\varepsilon\right)-(1$, $\left.\frac{4 \cdot \varphi \cdot \varepsilon}{1-\varphi+2 \cdot \varepsilon}, 0\right)-(1,1,0)$ from $\bar{P}_{2,2,2}\left(\frac{1+\varphi}{2}\right)=(0,1,0)-(0,0,0)-\left(\frac{1+\varphi}{2}, 0, \frac{1+\varphi}{2}\right)-\left(\frac{1}{2}, 1+\varphi\right.$, $\left.\frac{1}{2}\right)-\left(\frac{1-\varphi}{2}, 0, \frac{1+\varphi}{2}\right)-(1,0,0)-(1,1,0)$. Consequently, $\lim _{\varepsilon \rightarrow 0} \overline{\bar{P}}_{2,2,2}(\varepsilon)=\bar{P}_{2,2,2}\left(\frac{1+\varphi}{2}\right)$ (see Figure 9).

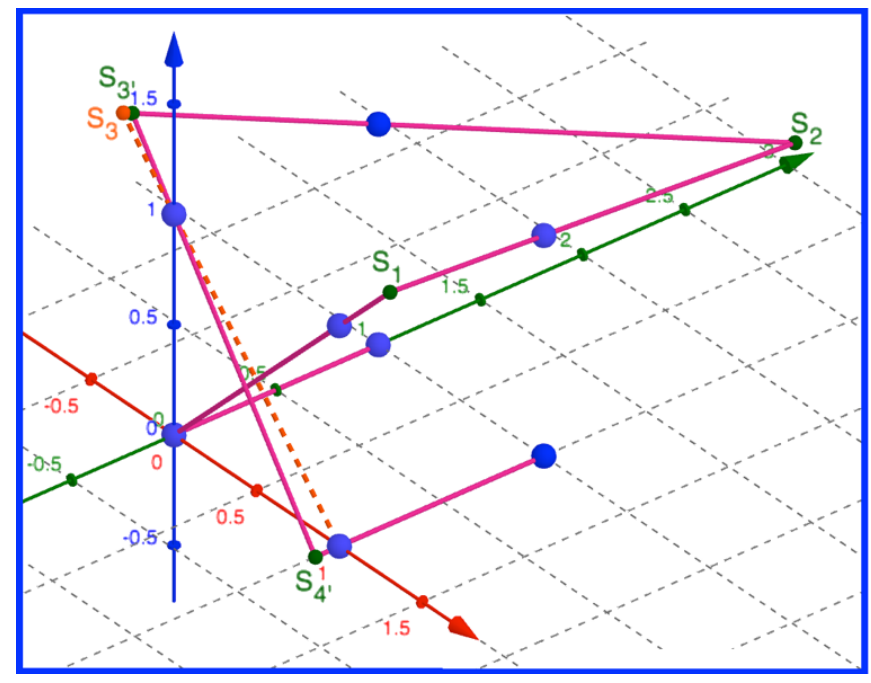

Figure 9. The self-crossing covering path $\bar{P}_{2,2,2}\left(\frac{1+\varphi}{2}\right)$ and the uncrossing covering path $\overline{\bar{P}}_{2,2,2}(\varepsilon)$, where the (Euclidean) distance between the point $(1,0,0)$ and the Steiner point $S_{4^{\prime}} \in \overline{\bar{P}}_{2,2,2}(\varepsilon)$ is given by $-y_{\mathrm{S}_{4^{\prime}}}=-\frac{4 \cdot \varphi \cdot \varepsilon}{1-\varphi+2 \cdot \varepsilon}$ (picture realized with GeoGebra). 
Therefore, $\overline{\bar{P}}_{2,2,2}(\varepsilon)$ shows the existence of minimal uncrossing covering paths which are entirely contained in $\left[\frac{1-\varphi}{2}+\varepsilon, \frac{1+\varphi}{2}\right] \times\left[\frac{4 \cdot \varphi \cdot \varepsilon}{1-\varphi+2 \cdot \varepsilon}, 1+\varphi\right] \times\left[0, \frac{1+\varphi}{2}\right] \subset[-0.309016$, $1.309017] \times[-10.47214 \cdot \varepsilon, 2.618034] \times[0,1.309017]$, an AABB $\nsubseteq$ RAABB with a volume of less than 5.5451 cubic units for any sufficiently small $\varepsilon$ (i.e., $\varepsilon \leq 1.1936 \cdot 10^{-7}$ will always work). Of course, this does not prove that $\overline{\bar{P}}_{2,2,2}\left(0^{+}\right)$is an optimal uncrossing covering path for the AABB volume, but constitutes a good example of the variety of hard open problems linked to any $G_{n_{1}, n_{2}, \ldots, n_{k}}$ grid, including the basic case $G_{2,2,2}$.

\section{CONCLUSION}

This paper ends the trilogy started with the introduction of the standard clockwise-algorithm [9], able to perfectly solve (with covering trails of minimal link length) the generalization to $k$ dimensions of Loyd's nine dots puzzle [10].

In the present work, we set a unique length class for every edge of any uncrossing covering path for $G_{n_{1}, n_{2}, \ldots, n_{k}}$, imposing also that the link length must be less than the number of points to be visited, and in addition, every covering path is constrained by a given AABB $\subseteq$ RAABB, so we studied different patterns according to the size of the AABB, with the purpose of reducing, for any $k>1$, the link length of the valid paths.

On the other hand, if we discard the RAABB constraint for our uncrossing covering path, even the minimization of the link length applied to $G_{2,2, \ldots, 2}$ turns into open problem for any $k \geq$ 3 (see Section IV), while a different kind of three-dimensional AABB, such as $\left[-\frac{1}{\sqrt{2}}, 1+\frac{1}{\sqrt{2}}\right] \times\left[0,1+\frac{1}{\sqrt{2}}\right] \times\left[0,1+\frac{1}{\sqrt{2}}\right] \nsubseteq$ RAABB (i.e., a box with a volume of only $\frac{(1+\sqrt{2})^{3}}{2}<7.035534$ units $^{3}$ but which is not entirely contained in the RAABB), let us speculate how $\left(-\frac{1}{\sqrt{2}}, 0,1+\frac{1}{\sqrt{2}}\right)-(1,0,0)-\left(-\frac{1}{\sqrt{2}}, 1+\frac{1}{\sqrt{2}}, 0\right)-\left(\frac{1}{2}, \frac{1}{2}, 1+\frac{1}{\sqrt{2}}\right)-\left(1+\frac{1}{\sqrt{2}}, 1+\frac{1}{\sqrt{2}}, 0\right)-$ $(0,0,0)-\left(1+\frac{1}{\sqrt{2}}, 0,1+\frac{1}{\sqrt{2}}\right)$ would solve the aforementioned problem if only we could allow self-intersecting for those 6 links of prescribed length $1+\sqrt{2}$ units.

Thanks to the $M \Lambda I$-algorithm, we showed that it is possible to provide acceptable covering paths, inside the MAABB, whose edges belong to the same integer (and not only irrational [3]) length class, in two, three, and more dimensions. We hope that the arising questions concerning how many valid length classes there are for any $k$-tuple $\left(n_{1}, n_{2}, \ldots, n_{k}\right)$, under many different sets of constraints [2] (even allowing trails, walks or trees [11] instead of merely paths), will provide a challenging environment for new researches in this subfield of graph theory, born from an ancient two-dimensional puzzle which was first published more than one hundred years ago [10].

Let us now conclude our research on this topic taking leave of us with a remarkable sentence by a revolutionary mathematician who died in the XIX century:

"Quand la concurrence, c'est-à-dire l'égoïsme, ne règnera plus dans la science, quand on s'associera pour étudier, au lieu d'envoyer aux académies des paquets cachetés, on s'empressera de publier ses moindres observations pour peu qu'elles soient nouvelles et en ajoutant : "Je ne sais pas le reste. »" Evariste Galois, De Ste Pélagie, December 1831 [12]. 
JOURNAL OF FUNDAMENTAL MATHEMATICS

AND APPLICATIONS (JFMA) VOL. 4 NO. 2 (NOV 2021)

Available online at www.jfma.math.fsm.undip.ac.id

\section{ACKNOWLEDGMENT}

We sincerely thank Koki Goma from Japan for having discovered and shared, in August 2021, the first known (to the best of our knowledge) unconstrained self-intersecting covering path for $G_{2,2,2}$ with 6 lines, allowing us to find the related covering paths which have been included in Section III, and inspiring also the covering circuits represented in Figures $7 \& 8$.

\section{REFERECES}

[1] P. Eades and N.C. Wormald, "Fixed edge-length graph drawing is NP-Hard", Discrete Applied Mathematics, vol. 28, pp. 111-134, 1990.

[2] S. Bereg, P. Bose, A. Dumitrescu, F. Hurtado and P. Valtr, "Traversing a Set of Points with a Minimum Number of Turns", Discrete \& Computational Geometry, vol. 41, no. 4, pp. 513-532, 2009.

[3] M. Ripà, "Reducing the clockwise-algorithm" to k length classes", Journal of Fundamental Mathematics and Applications, vol. 4, no. 1, pp. 61-68, 2021.

[4] S. Gravier and A. Parreau, "Hamiltonicité dans une grille, un jeu d'enfant ?", Internal report, École Normale Supérieure de Lyon, 2006. enslyon.free.fr/ rapports/info/Aline_Parreau_1.pdf

[5] M. Ripà, "Solving the $n \_1 \leq \mathrm{n} \_2 \leq \mathrm{n} \_3$ Points Problem for $\mathrm{n} \_3<6$, ResearchGate, 25 Jun. 2020. https://wWw.researchgate.net/publication/ 342331014_Solving_the_n_1_n_2_n_3_Points_Problem_for_n_3_6

[6] E. Kranakis, D. Krizanc and L. Meertens, "Link length of rectilinear Hamiltonian tours in grids", Ars Combinatoria, vol. 38, pp. 177-192, 1994.

[7] D.P. Wagner, "Path planning algorithms under the link-distance metric", Dartmouth College Ph.D. Dissertations, no. 16, 2006. https://digitalcommons.dartmouth.edu/dissertations/16/

[8] M. Hohenwarter, M. Borcherds, G. Ancsin, B. Bencze, M. Blossier, J. Elias, K. Frank, L. Gal, A. Hofstaetter, F. Jordan, B. Karacsony, Z. Konecny, Z. Kovacs, W. Kuellinger, E. Lettner, S. Lizelfelner, B. Parisse, C. Solyom-Gecse and M. Tomaschko, "GeoGebra - Dynamic Mathematics for Everyone - version 6.0.507.0-w", International GeoGebra Institute, 16 Oct. 2018. https : / / www . geogebra.org

[9] M. Ripà, "Solving the 106 years old $3^{\wedge} \mathrm{k}$ Points Problem with the clockwise-algorithm", Journal of Fundamental Mathematics and Applications, vol. 3, no. 2, pp. 84-97, 2020.

[10] S. Loyd, "Cyclopedia of Puzzles", The Lamb Publishing Company, New York, p. 301, 1914.

[11] A. Dumitrescu and C. Tóth, "Covering Grids by Trees", 26th Canadian Conference on Computational Geometry, 2014.

[12] J. Tannery, "Manuscrits de Évariste Galois / publiés par Jules Tannery", GauthierVillars, Paris, p. 27, 1908. 